\title{
Ich tat doch nur meine Pflicht! \\ Das Heroismus-Paradox der Supererogation
}

\section{I just did my simple duty \\ The Heroism-Paradox of Supererogation}

\author{
Marie-Luise Raters, Potsdam
}

„I don't consider myself a hero. Just a man who did his simple duty in opening his home to Jews“"

Staszek Jackowski nach dem Zweiten Weltkrieg, vgl. Tec 1986, 165

Zusammenfassung: Menschen, die moralisch Herausragendes getan haben, sagen mit signifikanter Häufigkeit, dass sie damit nur ,ihre Pflicht' getan hätten. So auch Staszek Jackowski, der im besetzten Polen 18 Monate lang 32 jüdische Menschen in einem Keller unter seinem Haus vor den Nazi-Verbrechern versteckt hatte. Der Essay diskutiert dieses irritierende Phänomen, das in der aktuellen angloamerikanischen Supererogationsforschung als ,Heroismus-Paradox ' bezeichnet wird. In einem ersten Schritt wird Jackowskis Handlungsweise als Supererogation (Ultraerogation) und damit als Nichtpflicht ausgewiesen. Anschließend werden verschiedene Lösungsvorschläge des Heroismus-Paradoxes diskutiert. Daraus wird ein alternativer Lösungsvorschlag entwickelt, demzufolge das Handeln von Supererogateuren in einer freiwilligen Selbstverpflichtung auf einen besonders anspruchsvollen Entwurf vom moralischen Selbst wurzelt. In zwei Ausblicken wird abschließend in grobem Umriss begründet, warum Mitläufer unanständig wirken und was unter Jackowskis Umständen tatsächlich moralische Pflicht gewesen wäre.

Schlagwörter: Supererogation, Pflicht, Heldentum, Widerstand, Heroismus-Paradox 
Abstract: People who have acted admirably often say 'I just did my simple duty' afterwards. An example is Staszek Jackowski: He hit 32 Jews from Nazis in a cellar under his house in Poland for 18 months. This paper discusses this puzzling phenomenon, which in current Anglo-American supererogation research is labeled as 'heroism paradox'. In a first step, I will show that Jackowski did not fulfil a duty, but his actions should be seen as supererogation (ultraerogation). In my second part I will discuss several solutions to the heroism paradox in the literature. Against this backdrop I will develop my solution that supererogatory actions derive from a voluntary self-obligation of the moral agent to a demanding vision of her future moral self. Finally, I will give two short ideas of why bystanders seem indecent and what actually would have been moral duty under Jackowski's circumstances.

Keywords: supererogation, duty/obligation, heroism, resistance, heroism-paradox

Im United States Holocaust Memorial Museum in Washington steht das Modell eines Hauses. Es bildet das Haus des polnischen Christen Staszek Jackowski nach, der seinen Keller ausgebaut hatte, um (zwei Blocks vom Gestapo-Hauptquartier entfernt) 32 jüdische Menschen für 18 Monate vor der Deportation durch die Nazi-Verbrecher zu verstecken. Jackowski war Automechaniker in der polnischen Stadt Stanisławów. Zunächst wollte er nur seinen jüdischen Sandkastenfreund Max Saginu mit dessen Frau, Schwager und Schwägerin retten, aber im Laufe der Monate kamen immer mehr hilfesuchende Menschen zu ihm, bis es schließlich 32 waren. ${ }^{1}$ Jackowski wusste, dass er zu Tode gefoltert worden wäre, wenn die Nazi-Verbrecher sein Treiben bemerkt hätten. Als er mit der Yad-Vashem-Medaille ausgezeichnet werden sollte, soll er jedoch gesagt haben: „I don't consider myself a hero. Just a man who did his simple duty in opening his home to Jews“" (Tec 1986, 165).

Mit diesem Diktum steht Jackoswki nicht allein: Tatsächlich lässt sich das erstaunliche Phänomen auffallend häufig beobachten, dass Menschen nach außergewöhnlichen moralischen Handlungen sagen, dass sie nur ihre Pflicht getan hätten. ${ }^{2}$ Damit wirft der ,Fall Jackowski` zwei generelle Fragen

$1 \quad$ Details finden sich in dem Interview Lukas 1989.

2 Pars pro toto seien einige genannt. (i) Baron führt in ihrem Essay Imperfect Duties and Supererogatory Acts von 1998 Beispiele aus Tecs Buch When Light Peirced the Darkness von 1986 an. So zitiert sie Ada Celka (die ein jüdisches Mädchen vor den Nazi-Verbrechern versteckt hatte) mit folgender Äußerung: „By saving the Jewish girl I simply did my duty. What I did was everybody's duty“ (Tec 1986, 165; zit. bei Baron 1998, 66). (ii) Archer und Ridge führen 
auf, die mein Essay in aller gebotenen Kürze behandeln soll. Die erste Frage lautet, ob Jackowski mit seiner Behauptung nicht schlicht recht gehabt haben könnte? War sein lebensgefährlicher Einsatz für die 32 Menschen tatsächlich seine moralische Pflicht? Mein Essay wird diese Frage verneinen und Jackowskis Handlungsweise als Supererogation ausweisen. Das wirft allerdings das Folgeproblem auf, warum Menschen wie Jackowski ihren Einsatz dennoch als ,Pflicht bezeichnen? Diese Frage wird in der aktuellen angloamerikanischen Supererogationsforschung unter dem Etikett ,Heroismus-Paradox ${ }^{6}$ (heroism-paradox ${ }^{3}$ ) diskutiert. Nach der Diskussion der beiden leitenden Fragen folgen zwei kurze Ausblicke. Zunächst wird knapp begründet, warum Mitläufer unanständig wirken. Der vierte Abschnitt skizziert im Umriss, was unter Jackowskis Umständen tatsächlich Pflicht gewesen wäre. ${ }^{4}$

\section{War Jackowskis Handeln tatsächlich Pflicht? In der Supererogations-} forschung werden Aussagen wie die von Jackowski gemeinhin von ,An-

das Beispiel des Unterleutnants Daniel K. Inouye ins Feld, der einen Orden erhalten hat, weil er im 2. Weltkrieg am 21. April 1945 im italienischen San Terenzo unter persönlicher Lebensgefahr 25 Feinde getötet und acht Gefangene gemacht hatte. In einem Interview soll Inouye Folgendes gesagt haben: „The pain was nothing great, and I had my job, my obligation and my mission to accomplish" (Archer und Ridge 2015, 1577; zit. nach Boghani 2012). (iii) Besonders häufig werden die Bewohner des französischen Dorfs Le Chambon erwähnt, die 5000 (!) jüdische Menschen vor den Nazi-Verbrechern versteckt haben. In Interviews sollen später immer wieder Äußerungen wie die folgende gemacht worden sein: „How can you call us ,good‘? We were doing what had to be done. Who else could help them?" (Hallie 1979, 20. Zit. u. a. in Hale 1991, 273f., sowie in Flescher 2000, 124ff.). (iv) Einem Artikel der Süddeutschen Zeitung zufolge soll der 24-jährige islamische Geflüchtete Lassana Bathily ein Buch mit dem Titel Ich bin kein Held geschrieben haben, nachdem er während eines islamistischen Terroranschlags auf einen jüdischen Supermarkt im Osten von Paris unter Einsatz seines Lebens mehrere Kunden vor dem Terroristen versteckt hatte (Pantel 2018, 7).

3 Das Etikett ,Heroismus-Paradoxon w wurde meiner Rekonstruktion zufolge geprägt in Archer/Ridge 2015, 1577. Diskutiert werden (scheinbar) widersprüchliche Aussagen von Supererogateuren. Deshalb ist das HeroismusParadox nicht identisch mit dem metaethischen Problem des moralischen Werts (s.u. Anm. 8).

$4 \quad$ Aufrichtig bedanken möchte ich mich bei den beiden Gutachter(inne)n der Zeitschrift für Praktische Philosophie für ihre wertvollen Verbesserungsvorschläge. 
ti-Supererogationisten ${ }^{5}$ zitiert, um davon zu überzeugen, dass es keine Supererogationen gibt. So heißt es bei Susan Hale: „Since all morally good acts are required, saints and heroes are right when they say (...) that they have only done their duties" (Hale 1991, 279). ${ }^{6}$ Im Hintergrund steht das metaethische Argument des moralischen Werts, dem zufolge alle moralisch wertvollen Handlungen unabhängig von den Kosten für die moralischen Akteure allgemeine Pflicht sein müssen, weil sie moralisch wertvoll sind.7 Moralisch wertvolle Handlungen, die keine Pflicht sind, kann es diesem Argument zufolge nicht geben. Damit betrifft das anti-supererogationistische Argument zwar den Kern jeder Theorie der Supererogation, weil es keine Supererogationen geben kann, wenn ausnahmslos jede moralisch wertvolle Handlung Pflicht ist. Dennoch muss an dieser Stelle eine thetische Widerlegung genügen.

(1.1) Mit dem Argument werden Handlungen wegen ihres moralischen Werts als Pflicht behauptet. Deshalb müssen zunächst im groben Umriss ${ }^{8}$ die Begriffe ,moralischer Wert' und ,Pflicht' geklärt werden. Von einer , allgemeinen moralischen Pflicht' sei die Rede, wenn ein moralischer Akteur ${ }^{9}$ vor dem

$5 \quad$ Es hat sich in der angloamerikanischen Supererogationsforschung eingebürgert, diejenigen Theoretiker als ,Supererogationisten“ (supererogationists) zu bezeichnen, die eine eigenständige Kategorie supererogativer Handlungen befürworten; ,Anti-Supererogationisten' heißen die Theoretiker, die das ablehnen. Zur Abgrenzung sollten moralische Akteure, die supererogativ handeln, als ,Supererogateure“ bezeichnet werden.

6 Vgl. mit derselben Stoßrichtung auch New 1974, 184 sowie Baron 1987, 252.

7 Walter Pfannkuche hat das Argument so formuliert: „Wer anerkennt, dass eine Handlung auch für seine Situation moralisch richtig ist oder moralischen Wert hat, und ein moralischer Mensch sein will und in der Lage ist, die Handlung auszuführen, der muss auch anerkennen, dass er so handeln soll und sich Vorwürfe machen, falls er es nicht tut“ (Pfannkuche 1994, 79). Vgl. auch Pybus 1982, 195. Obwohl in der Supererogationsforschung mehrere (scheinbare) Paradoxien diskutiert werden, wird das Problem des moralischen Wert mancherorts auch als ,das Paradox der Supererogation“ bezeichnet. Vgl. z. B. Horgan/Timmons 2010.

8 Detaillierte Herleitungen und Verteidigungen meiner Theorie der Supererogation erfolgen in einem Buch, das ich gerade fertigzustellen versuche. Vgl. dazu Raters: Unvollendetes Typoskript.

9 Gemeint ist die geschlechtsneutrale Rollenbezeichnung: Es sind also Akteurinnen und Akteure gemeint. 
Hintergrund des kollektiven Normengefüges seiner Zeit vernünftigerweise wollen kann, dass alle moralischen Akteure unter vergleichbaren Umständen die Handlung $x$ tun sollten. Diesem rationalistischen Pflicht-Begriff ${ }^{10}$ zufolge muss ein Akteur vernünftige Gründe haben, wenn er eine Handlung als allgemeine moralische Pflicht behaupten will, wobei ,vernünftige Gründe solche sind, die anderen Akteuren rational verständlich und einsichtig sind. Dass die kollektiven Normengefüge einer Zeit Berücksichtigung finden sollen, basiert auf den pragmatistischen Überzeugungen, dass sich unsere Lebenswirklichkeit (durch technische Innovationen oder soziale Umwälzungen beispielsweise) ständig wandelt und dass sich unsere Systeme von Pflichten den sich wandelnden Lebenswelten anpassen müssen, damit die Moral nicht zum rigoristischen Selbstzweck verkrustet. Vom ,moralischen Wert einer Handlung spreche ich, wenn eine Handlung $x$ die berechtigten Interessen ${ }^{11}$ von anderen moralischen Akteuren erstens befördern soll (Intention bzw. Absicht) und zweitens auch tatsächlich befördert (Folgen für andere) und ihr schließlich auch ein gutes Motiv (Beweggrund) zugrunde liegt. Handlungen ohne gute Folgen für andere sind offensichtlich nicht moralisch wertvoll, weil man nicht wollen würde, dass sie wiederholt werden. Dass eine Handlung ohne gute Absichten nicht moralisch wertvoll ist, zeigt sich daran, dass wir uns nicht bedanken würden, wenn jemand uns etwas Gutes tut, ohne uns etwas Gutes tun zu wollen. Dass eine Handlung ohne gutes Motiv keine gute Handlung ist, wird schließlich daraus ersichtlich, dass wir uns betrogen fühlen, wenn uns jemand mit schlechtem Motiv etwas Gutes tut. Inkompetente Hilfeleistung wäre demnach keine moralisch wertvolle Handlung, weil allen guten Absichten zum Trotz die berechtigten Interessen anderer nicht befördert werden; ebenfalls nicht moralisch wertvoll wäre es, wenn sich jemand für einen anderen nur opfert, um den anderen zu beschämen, weil dann keine gute Intention zugrunde liegt; dasselbe gilt schließlich auch für Liebesdienerei, weil kein moralisch wertvolles Motiv zugrunde liegt. ${ }^{12}$

$10 \quad$ Im Hintergrund steht die Auffassung von ,Pflicht‘ in Hare 1981/1992.

11 Zur Abgrenzung von, berechtigten“ Interessen von ,banalen“ oder ,bösen“ Interessen vgl. Hare 1981/1992, 203-206.

12 Auf den komplizierten Fall der gut intendierten Handlung, die wegen nicht absehbarer böser Zufälle keine guten Folgen für andere hat, kann ich hier nicht eingehen. Nur so viel: Das moralische Urteil über die Handlung ist vom moralischen Urteil über den Akteur zu unterscheiden. Eine durch nicht absehbare böse Zufälle gescheiterte Handlung wäre nicht moralisch wertvoll, 
(1.2) Der moralische Wert einer Handlung ist nun tatsächlich ein guter Grund zu wollen, dass die Handlung allgemeine Pflicht sein sollte. So viel sei dem Argument des moralischen Werts zugestanden. Allerdings kann der moralische Wert einer Handlung nur eine notwendige, aber keine hinreichende Bedingung dafür sein, weil es bei manchen moralisch wertvollen Handlungen Gründe gibt, aus denen man trotz ihres moralischen Werts dennoch nicht wollen kann, dass sie allgemeine Pflicht sein sollen. Wenn solche Gründe vorliegen, kann eine Handlung keine allgemeine Pflicht sein: Sie wäre Supererogation. Damit ist eine ,Supererogation' eine moralisch wertvolle Handlung $x$, bei der ein moralischer Akteur trotz ihres moralischen Werts aus vernünftigen Gründen nicht wollen kann, dass die Handlung allgemeine Pflicht sein sollte.

(1.3) Kompliziert wird die Angelegenheit dadurch, dass es zwei Arten solcher Gründe zu geben scheint, weshalb man zwei Arten von Supererogationen unterscheiden muss. Wenn man nicht wollen kann, dass eine moralisch wertvolle Handlung allgemeine Pflicht ist, weil sie den moralischen Akteur als solchen gefährden würde, spreche ich von einer Ultraerogation. Zur Frage, warum man das vernünftigerweise nicht wollen kann, wird unten noch einiges zu sagen sein (vgl. Abschnitt 1.4). Hier ist festzuhalten, dass Rettungsaktionen, die das Leben des Retters gefährden, Ultraerogationen sind. ${ }^{13}$ Jenseits der Gefährdung des moralischen Akteurs als solchen gibt es

weil die Handlung ihr Ziel nicht erreicht hat. Dennoch wäre aber der Akteur moralisch lobenswert, weil man sich für die gute Absicht bedanken würde. Das wiederum tun wir, weil wir von der guten Absicht in aller Regel auf ein gutes Motiv zurückschließen können. Vgl. zu den Problemen einer trennscharfen Unterscheidung zwischen Motiven und Absichten von Supererogationen Benn 2019.

13 Dasselbe gilt für moralisch wertvolle Handlungen, mit denen ein Akteur seinen Lebensinhalt, seinen Lebensentwurf oder seine Autonomie gefährdet, um ohne Anspruch auf Vollständigkeit einige weitere Gründe für Ultraerogationen zu nennen. Weil im vorliegenden Kontext (vor allem) der Grund der Gefährdung des Lebens einschlägig ist, möchte ich zu den übrigen möglichen Gründen für Ultraerogationen nur knappe Anmerkungen machen. (i) Der ,Lebensinhalt‘ ist dasjenige, was das Leben eines Menschen in einem substantiellen Sinne sinnvoll und glücklich macht. Insofern handelt jemand ultraerogativ, der in seiner Ehe seinen Lebensinhalt sieht, aber dennoch gegen den Willen des Ehepartners zu, Ärzte ohne Grenzen' geht, obgleich das die Auflösung seiner Ehe bedeutet. (ii) Unter dem ,Lebensentwurf" verstehe ich im Sinne von Jean-Paul Sartre den Plan, den jemand von sich selbst ge- 
nun noch andere Gründe, aus denen man bei manchen moralisch wertvollen Handlungen nicht wollen kann, dass sie allgemeine Pflicht sind: Dann spreche ich von Anstandserogationen. ${ }^{14}$ Ein solcher Grund kann beispielsweise die pragmatische Unverhältnismäßigkeit des Handlungsaufwandes im Vergleich zum Nutzen für den Begünstigten sein, aber auch bestimmte Zuständigkeitsregelungen beispielsweise. Weil Jackowskis Handlungsweise eindeutig keine Anstandserogation war, möchte ich auf diese Spielart der Supererogationen an dieser Stelle jedoch nicht weiter eingehen. ${ }^{15}$

macht hat (vgl. Abschnitt 2.4). Weil Nierenspender keine Leistungssportler mehr sein können, handelt ein Leistungssportler mit der Lebendspende einer Niere ultraerogativ. (iii) Ein weiterer Kandidat wäre eine Handlung, die die Autonomie oder die Handlungsfähigkeit gefährden könnte. Somit handelt jemand ultraerogativ, der einen Angehörigen so lange pflegt, bis er selbst Hilfe braucht. (iv) Ein Suizidversuch aus einer Depression heraus ist hingegen keine Ultraerogation, weil eine solche Handlung nicht moralisch wertvoll ist. Aus demselben Grund ist es nicht ultraerogativ, ein Verbrechen zu begehen, um einem anderen Menschen zu helfen. Falls das Verbrechen die einzige Möglichkeit zur Hilfe wäre, läge vielmehr ein Dilemma vor. Das zu begründen, würde hier jedoch zu weit führen.

14 Ohne Weiteres zugeben möchte ich den naheliegenden Einwand, dass die Grenze zwischen Anstands- und Ultraerogationen nicht immer so distinkt gezogen werden kann, wie es in der Praxis wünschenswert wäre, weil Anstandserogationen durch Häufung oder zu lange Dauer in Ultraerogationen umschlagen können. Hervorzuheben ist jedoch, dass sich die Unterscheidung nicht auf eine Unterscheidung zwischen heroischen oder heiligmäßigen Akten gegenüber banalen Gefälligkeiten reduzieren lässt. Vgl. dazu Raters 2020 (i. E.).

Vgl. ausführlich Raters: Unvollendetes Typoskript. (i) Von ,pragmatischer Unverhältnismäßigkeit‘ spreche ich, wenn der Gewinn der Handlung für den Begünstigten deutlich geringer ist als der Aufwand für den moralischen Akteur. (ii) Weil es alle Zuständigkeitsregelungen überflüssig machen würde, wenn ein Handeln ohne Zuständigkeit allgemeine Pflicht wäre, kann man das vernünftigerweise nicht wollen. (iii) Ein weiterer Grund für die Diagnose ,Anstandserogation “ kann der ,ästhetische Überschuss' einer Handlung sein. Diesen weisen Handlungen auf, mit denen man besondere Sympathie bekunden oder Dankbarkeit zeigen kann. Wenn Gefälligkeiten und Freundschaftsdienste Pflicht wären, gäbe es keinen Raum für spezielle Sympathiebekundungen mehr, und es würde sich niemand mehr bedanken müssen. Beides wäre ein ästhetischer Verlust, den man vernünftigerweise nicht wollen kann. Obgleich man sagen kann, dass man jemandem ,einen Gefallen schuldet', sind Gefälligkeiten deshalb ebenso wie Freundschaftsdienste Anstandserogationen. 
(1.4) Jackowskis Handlungsweise war moralisch wertvoll, weil sie viele Menschenleben gerettet hat. Vor allem aber war sie lebensgefährlich: Jackowski wäre zu Tode gefoltert worden, wenn die Gestapo ihn erwischt hätte. Das bedeutet, dass Jackowskis Handlungsweise ultraerogativ war, weil sich Jackowski mit seinem moralisch wertvollen Handeln als moralischer Akteur selbst in seinem Status als moralischer Akteur gefährdet hat. Dieser Diagnose würde ein Antisupererogationist wie New allerdings entgegenhalten, dass es selbstverständlich die Pflicht gibt, sich für andere opfern zu müssen. ${ }^{16}$ (i) Wer eine solche Pflicht behauptet, begeht jedoch erstens eine Art utilitaristischen Rechenfehler. Wenn ein moralischer Akteur sich selbst als solchen opfert, verschwindet aus der Perspektive seines subjektiven Erlebens seine ganze Welt mit ihm. Deshalb ist ein Selbstopfer aus der Sicht des Akteurs eine absolute Größe, die man als solche in keine utilitaristische Gesamtnutzenabwägung sinnvoll einbringen kann. (ii) Zweitens würden moralische Akteure für die Zwecke von anderen Akteuren in unzulässiger Weise instrumentalisiert. (iii) Vor allem aber wäre es widersprüchlich, wenn im Namen einer Moral gefordert würde, dass sich moralische Akteure für andere moralische Akteure opfern müssen. Die Moral dient dem Schutz der berechtigten Interessen von moralischen Akteuren. ${ }^{17}$ Nun ist der Akteur, der sich opfern müsste, selbst ein moralischer Akteur. Wenn man diese beiden Prämissen akzeptiert (und ich denke, dass man sie akzeptieren sollte), würde es zu einem Selbstwiderspruch der Moral führen, wenn es eine allgemeine Pflicht für moralische Akteure gäbe, sich als moralische Akteure für ande-

(iv) Von den ,usurpatorischen Tendenzen der Moral' spreche ich, weil wir ununterbrochen moralisch wertvoll handeln müssten, wenn alles moralisch Wertvolle Pflicht wäre, das wir tun könnten, weil es immer etwas moralisch Wertvolles zu tun gibt. Auch das kann man vernünftigerweise nicht wollen. Deshalb können die usurpatorischen Tendenzen der Moral ein vernünftiger Grund sein, nicht zu wollen, dass alle moralisch wertvollen Handlungen allgemeine Pflicht sind. (v) Als Pseudo-Anstandserogationen auszuschließen sind unfähige Hilfe, Amtsanmaßungen, Helfersyndrome und Anbiederungen beispielsweise, weil solche Handlungen nicht moralisch wertvoll sind.

16 Selbstopfer moralischer Akteure für andere Akteure sind nach New sogar dann moralische Pflicht, wenn das Gesamtwohl nicht maximiert wird (New 1974, 185).

17 Dazu heißt es schon in Urmsons Essay Saints and Heroes von 1958: „Morality, as I take it, is something that should serve human needs“ (Urmson 1958, 210). 
re moralische Akteure opfern zu müssen. Was selbstwidersprüchlich ist, ist unvernünftig, ${ }^{18}$ was unvernünftig ist, kann man vernünftigerweise nicht als allgemeine Pflicht wollen; somit kann es keine allgemeine Pflicht zu Handlungen geben, durch die sich moralische Akteure als moralische Akteure selbst opfern müssen. ${ }^{19}$ Wenn jemand wie Jackowski dennoch sein Leben für andere gefährdet, handelt er demnach ultraerogativ. Das ist der Kern meines Arguments.

2. Warum hat Jackowski gesagt, er habe nur seine Pflicht getan? Jackowskis Handlungsweise war trotz ihres moralischen Werts wegen ihrer Lebensgefährlichkeit keine allgemeine Pflicht, sondern Ultraerogation. Mit dieser Diagnose stellt sich die weiterführende Frage, warum Jackowski dann trotzdem gesagt hat, dass er ,nur seine Pflicht' getan habe? In der angloamerikanischen Supererogationsforschung werden unterschiedliche Lösungsvorschläge für dieses ,Heroismus-Paradox ' diskutiert.

(2.1) Manche Autoren erklären die Äußerungen als Ausdruck von Bescheidenheit. $\mathrm{Zu}$ unterscheiden sind falsche ${ }^{20}$ und echte ${ }^{21}$ Bescheidenheit. (i) Wer Bescheidenheit heuchelt, will eigentlich sagen, dass die außergewöhnliche Handlung für ihn quasi normal und alltäglich sei, weil er selbst außergewöhnlich sei. Deshalb würde er andere Situationen erwähnen, in denen er ebenfalls außergewöhnlich gehandelt hat. Nach allem, was über ihn geschrieben wurde, hat Jackowski das aber nie getan. (ii) Er soll im Gegenteil in einem Interview von 1989 sogar Folgendes gesagt haben: „I have been

18 Um einem möglichen Missverständnis vorzubeugen: Mit dem Argument, dass man vernünftigerweise das Selbstopfer moralischer Akteure für andere Akteure nicht als allgemeine Pflicht wollen kann, ist ausdrücklich nicht gesagt, dass es per se irrational ist, sich für andere zu opfern. Es kann im Gegenteil sehr rationale Gründe geben, das zu tun, wobei es sich allerdings um nicht verallgemeinerbare Gründe handeln wird. So kann es beispielsweise rational sein, sich für jemanden zu opfern, den man sehr liebt.

19 Das gilt auch für Soldaten: Fahnenflüchtige Soldaten verletzen ihre Pflicht, zu kämpfen, aber nicht die Pflicht, ihr Leben zu opfern, weil es eine solche Pflicht nicht geben kann. Dementsprechend verbietet unser Militärgesetz alle Todeskommandos. Den Hinweis verdanke ich Dieter Birnbacher.

20 Falsche Bescheidenheit vermutet beispielsweise Urmson 1958, 203.

21 New deutet diese Möglichkeit im Nebensatz an, wenn er schreibt: „Saints and heroes often deflect praise with the disclaimer ,I was only doing my duty. I conclude that they are, though modest, right“" (New 1974, 184). 
called a hero because I saved the lives of thirty-two Jewish men, women and children in Stanistawów. I don't consider myself a hero; many other Poles also helped Jews“ (Lukas 1989, 77). Es waren zwar nicht ,many other Poles', die Juden geholfen haben. Als der Krieg vorbei waren, haben von den 25.000 Juden aus Stanistawów nur 100 in Verstecken überlebt, und davon immerhin 32 in Jackowskis Bunkern (Sturrock 2011, o.S.). Aber dennoch legt Jackowski wohl tatsächlich echte Bescheidenheit an den Tag, wenn er betont, dass er nicht der Einzige in seiner Heimatstadt gewesen sei, der jüdische Menschen gerettet habe. Insofern könnte auch seine Äußerung zum Pflichtcharakter seines lebensgefährlichen Einsatzes von echter Bescheidenheit getragen gewesen sein. Allerdings ist ,Bescheidenheit‘ eine Charaktereigenschaft und keine Eigenschaft von Handlungen. Deshalb sagt die Bescheidenheit des Menschen Jackowski nichts über die hier strittige Frage aus, ob seine Handlungsweise allgemeine Pflicht war. ${ }^{22}$

(2.2) Jackowski könnte sich natürlich auch schlicht geirrt haben: Laut Susan Hale lautet so die ,Standard-Erklärung' des Heroimus-Paradoxons in der aktuellen Supererogationsforschung (Hale 1991, 279). Dabei sind nun drei Arten von Irrtümern zu unterscheiden. (i) In der Supererogationsforschung wird häufig die Möglichkeit erwogen, dass die Akteure (aus Zeitdruck oder wegen mangelnder intellektueller Fähigkeiten) nicht zu einer rationalen Abwägung über die Grenzen ihrer Pflicht in der Lage gewesen sein könnten (Curtis 1981, 317). Für Jackowski kann das definitiv ausgeschlossen werden: Dazu hat er viel zu ausdauernd und kontrolliert gehandelt, als er über 18 Monate hinweg die Bunkeranlagen unter seinem Haus immer mehr erweiterte und 32 Menschen mit Lebensmitteln, Strom und Wasser versorgte (Lukas 1989, 77f.). (ii) Dass Jackowski die Lebensgefährlichkeit seines Handelns falsch eingeschätzt haben könnte, kann ebenfalls ausgeschlossen werden. Auf die Frage, warum er im Laufe der Monate immer mehr Menschen bei sich aufgenommen hat, soll er seiner späteren Ehefrau ${ }^{23}$ Joanne nämlich geantwortet haben „They can only kill me once“ (Sturrock 2011, o.S.). So

22 Mit diesem Argument betont Jackson, dass ein Akteur kein ,moralphilosophisches Statement' (ethical statement) zu bestimmten Handlungstypen ablege, wenn er aus echter Bescheidenheit sagt, dass er mit seinem supererogativen Handeln ,nur seine Pflicht' getan habe (Jackson 1986, 292).

23 Um eine naheliegende Nachfrage zu antizipieren: Jackowski hatte zur fraglichen Zeit noch keine eigene Familie, die er mit seinem Handeln hätte gefährden können. Seine Frau Joanne heiratete er erst im Jahr 1946 in den USA, wo dann auch seine drei Söhne zur Welt kamen. Vgl. Gruber 1983. 
lapidar der Satz auch klingen mag, so zeugt er doch deutlich davon, dass sich Jackowski über die möglichen Folgen seines Handelns voll im Klaren war. (iii) Schließlich könnte sich Jackowski über die tatsächlichen Grenzen seiner Pflicht geirrt haben, weil er eine zu strenge Moral hatte. ${ }^{24}$ Für diese Möglichkeit spricht, dass Jackowski in einem Interview von 1989 gesagt hat: „I did what I had to do. After all Jews were human beings“" (Lukas 1989, 77). Diese Sätze können als moralphilosophisches Statement über eine allgemeine Nothilfepflicht gegenüber allen Menschen gelesen werden. Gegen diese Interpretation spricht jedoch, dass Jackowski den Polen Bogdanowicz ausdrücklich mit dem Hinweis auf die Lebensgefährlichkeit seiner Situation entschuldigt hat. Bogdanowicz hatte ursprünglich ebenfalls bedrohte Menschen aufgenommen, aber sie dann zu Jackowski geschickt, weil seine Angst übermächtig geworden war. Das hat Jackowski im Nachhinein folgendermaßen entschuldigt: „Bogdanowicz was afraid to keep his Jews any longer for fear of getting caught by the Gestapo. After all, any Pole caught helping a Jew was automatically executed“ (Lukas 1989, 77). Hervorzuheben ist, dass Jackowski ausdrücklich nicht von einer Pflichtverletzung Bogdanowiczs spricht! Er sagt im Gegenteil, dass Bogdanowiczs Angst ein nachvollziehbarer Grund gewesen sei, die Menschen nicht mehr verstecken zu wollen. Obwohl Jackowski den Begriff, Ultraerogation ' (natürlich) nicht verwendet hat, hat er damit der Sache nach nichts anderes gesagt, als dass das Verstecken von Juden unter den gegebenen lebensgefährlichen Umständen keine Pflicht, sondern Ultraerogation war.

(2.3) Jackowskis Verteidigung von Bodganowicz könnte auch nahelegen, dass er an sich selbst strengere moralische Maßstäbe angelegt haben könnte als an andere, weil er geglaubt hat, moralisch besonders berufen oder mit besonderen Fähigkeiten ausgestattet zu sein. So könnte sich Jackowski beispielsweise für besonders mutig oder für besonders mitleidig gegenüber dem Leiden anderer Menschen gehalten haben. Deshalb könnte er sich verpflichtet gefühlt haben, sich besonders anspruchsvolle moralische Prinzipien zu eigen zu machen, nach denen zu handeln er den ,normalen moralischen Akteuren nicht zutrauen würde. Mit seinem Diktum ,Ich habe nur meine Pflicht getan ' hätte er dann etwas ausgesprochen, was tatsächlich der Fall gewesen wäre, weil er sich wegen seiner besonderen Fähigkeiten ja Handlungen zur Pflicht gemacht hätte, von denen er glaubte, dass sie andere moralische Akteure überfordern würden. Mit dieser Erklärung könnte 
man sich auf R.M. Hare berufen, dem zufolge man sich besondere moralische Standards setzen sollte, wenn man glaubt, über besondere moralische Fähigkeiten zu verfügen: Veranschaulichend schreibt Hare ${ }^{25}$ von sich selbst, dass er „weit anspruchsvollere Prinzipien“ hätte, wenn er „so heiligmäßig wie Albert Schweitzer oder Mutter Theresa wäre“ (Hare 1981, 271). Im vorliegenden Zusammenhang weniger wichtig ist vielleicht der metamoralische Einwand, dass moralische Ausnahmemenschen moralphilosophisch eher uninteressant sind, weil sie als Ausnahmemenschen kein Leitbild ${ }^{26}$ für ,normale“ menschliche Akteure sein können. Wichtig ist jedoch, dass es den unsympathischen Beigeschmack von moralischem Hochmut hätte, wenn eine Ultraerogateur mit der scheinbar so demütigen Äußerung, er habe nur seine Pflicht getan, tatsächlich sagen will, dass er in moralischer Hinsicht etwas Besonderes sei. Es wirkt unsympathisch und vielleicht sogar unanständig, wenn jemand von sich selbst behauptet, besonders heldenhaft oder (um noch einmal mit Hare zu sprechen) besonders „heiligmäßig“ zu sein. Glücklicherweise kann das im Falle von Jackowski ebenfalls ausgeschlossen werden angesichts seiner schon zitierten Äußerung, dass „many other Poles also helped Jews“ (Lukas 1989, 77). Schließlich betont er damit ja, dass er nicht heldenhafter als ,many other Poles' gehandelt habe: Als Ausnahmemensch hat er sich also definitiv nicht gesehen.

(2.4) Damit bleibt nur noch die Möglichkeit, dass sich Jackowski in moralischer Hinsicht mehr abverlangt haben könnte als anderen, weil er sich vorgenommen hat, in moralischer Hinsicht ein besonders guter Mensch werden zu wollen. Um plausibilisieren zu können, warum sich so das Heroismus-Paradox lösen lässt, muss ich ein wenig ausholen. (i) Laut Jean-Paul Sartre ist es für alle vernünftigen Menschen kennzeichnend, dass sie einen Entwurf von ihrem künftigen Selbst machen und im Handeln zu realisieren versuchen. ${ }^{27}$ Menschen planen, wer sie einmal werden wollen, und ver-

$25 \quad$ Hare greift damit einen Vorschlag von Urmson 1958 auf. Mit Hare wird das Heroismus-Paradox erklärt in Witschen 2006, 2.

26 Flescher betont (gegen Urmson u. a.), dass ,Heilige und Helden' ganz normale (ordinary) moralische Akteure seien, die ihren Sinn für die Pflicht (sense of duty bzw. sense of love) besonders ausgeprägt hätten (Flescher 2000, 22-38).

27 Bei Jean-Paul Sartre heißt es dazu, dass der Mensch anders als „ein Blumenkohl zuerst ein Entwurf" von sich sei, den er in seinem Handeln zu realisieren versucht, um irgendwann „der Mensch“ zu sein, „der zu sein geplant hat“ 
suchen dann, den Plan zu realisieren. Dass solche Entscheidungen in aller Regel vom gesellschaftlichen Kontext abhängig sind, ist hier ebenso wenig von Interesse wie die Tatsache, dass die Entscheidung für einen bestimmten Selbstentwurf oft unbewusst gefällt wird. Wichtig ist jedoch, dass Selbstentwürfe immer auch eine moralische Dimension haben. Der Entwurf vom zukünftigen Selbst impliziert immer auch eine Vorstellung davon, welchen Stellenwert moralisches Handeln haben soll. (ii) Es gibt nun anspruchsvolle und weniger anspruchsvolle moralische Selbstentwürfe. Supererogateure sind Menschen, die sich für ein besonders anspruchsvolles moralisches Selbstbild entschieden haben. Die Gründe für eine solche Entscheidung können so vielfältig sein wie die Selbstentwürfe selbst. Im Falle Jackowskis mag sein christlicher Glaube eine zentrale Rolle gespielt haben. In der Berliner Ausstellung Topographie des Terrors findet sich eine Tafel mit folgender Äußerung des Christen Nikolaus Groß:28 „Wenn wir heute nicht unser Leben einsetzen, wie sollen wir dann vor Gott und unserem Gewissen bestehen?“ (Groß 1944, o.S.). Vielleicht hat Jackowski aber auch als polnischer Patriot oder als Humanist gehandelt; darüber lässt sich nur spekulieren. Festzuhalten ist jedoch, dass sich Jackowski offensichtlich irgendwann bewusst oder unbewusst entschieden hat, ein Mensch sein zu wollen, der andere Menschen sogar dann noch vor Folter und Tod beschützt, wenn das lebensgefährlich ist. Eine solche Entscheidung ist zweifellos eine Entscheidung für einen besonders anspruchsvollen (ultraerogativen) Entwurf vom moralischen Selbst. (iii) Hervorzuheben ist auch, dass eine solche Entscheidung nur eine freiwillige Selbstverpflichtung sein kann, weil es zwar eine allgemeine Metapflicht geben mag, seine Pflicht tun zu müssen, aber keine allgemeine Metapflicht, einen besonders anspruchsvollen Entwurf vom zukünftigen moralischen Selbst haben zu müssen. Wie Richard M. Hare treffend betont, ist in jeder moralischen „,sollte-Aussage implizit ein Prinzip enthalten, demzufolge die Aussage auf alle genau ähnlichen Situationen anwendbar ist“ (Hare 1991,

(Sartre 1946/1973, 11). Sartres Position beruht auf der atheistischen Prämisse, dass es die menschliche Seele im Sinne eines von Gott geschaffenen ,Bauplans` des Menschen nicht gibt. Für eine Diskussion dieser Prämisse ist hier nicht der Ort.

28 Der Bergmann und bekennende Christ Nikolaus Groß wurde nach dem Attentat vom 20. Juli 1944 wegen seiner Verbindungen zu Carl Goerdeler am 23. Januar 1945 in Berlin-Plötzensee ermordet. Vgl. https://www.gdw-berlin.de/vertiefung/biografien/personenverzeichnis/biografie/view-bio/nikolaus-gross/?no cache=1. Zugriff: 16. August 2018. 
41). Ein ,besonders' anspruchsvoller moralischer Selbstentwurf hebt sich nun im komparativischen Sinne von ,normalen“ moralischen Selbstentwürfen ab. Man kann also schon deshalb nicht sinnvoll fordern, dass alle moralischen Akteure einen ,besonders' anspruchsvollen moralischen Selbstentwurf haben sollen, weil sich durch ein solches ,Sollen` alle Selbstentwürfe so weit angleichen würden, dass es ,besonders' anspruchsvolle moralische Selbstentwürfe nicht geben könnte. Weil eine allgemeine Metapflicht zu einem besonders anspruchsvollen moralischen Selbstentwurf sinnlos wäre, muss es sich um eine freiwillige ${ }^{29}$ Selbstverpflichtung handeln. (iv) Weil sich moralische Selbstentwürfe ausschließlich durch entsprechendes Handeln realisieren lassen, müssen sich Supererogateure nicht nur freiwillig auf einen anspruchsvollen Entwurf vom moralischen Selbst entschieden haben, sondern sie müssen zudem auch bereit sein, für die Veredlung ihres moralischen Selbst (d.h. für die Realisierung ihres anspruchsvollen moralischen Selbstentwurfs) zu handeln, sobald sich die Gelegenheit dazu ergibt. Auch das hat Jackowski getan, als er die hilfesuchenden Menschen über Monate hinweg bei sich versteckt hat. (v) Weil offensichtlich kein Eigeninteresse vorlag und das Motiv der Pflichterfüllung ebenso ausgeschlossen werden konnte wie die Möglichkeiten eines Irrtums oder einer arroganten Selbstbeweihräucherung, kann aus Jackowskis Handeln also insgesamt nur geschlossen werden, dass er wegen einer freiwilligen Selbstverpflichtung auf einen besonders anspruchsvollen moralischen Selbstentwurf supererogativ (genauer: ultraerogativ) gehandelt hat.

(2.5) Was für Jackowski gilt, lässt sich verallgemeinern: Supererogateure sind moralische Akteure, die eine freiwillige Selbstverpflichtung auf einen besonders anspruchsvollen moralischen Selbstentwurf eingegangen sind und dann auch für die Veredelung ihres moralischen Selbst handeln. Das wiederum bedeutet, dass es sich beim sogenannten Heroismus-Paradox gar nicht um ein ,Paradox‘ handelt, sondern um eine Äquivokation im Pflichtbegriff. Es wäre tatsächlich paradox (bzw. selbstwidersprüchlich), wenn Jackowski seine Rettung der 32 Menschen als allgemeine Pflicht behauptet hätte, um dann gleichzeitig Bogdanowicz mit dem Argument der Supererogation zu entschuldigen, dass eine Rettung unter den gegebenen Umständen lebensgefährlich und deshalb keine Pflicht war. Es ist aber nicht

29 Vgl. zur Freiwilligkeit der Festlegung auf anspruchsvolle moralische Ideale auch Stemmer 2000, 310-315. Die entgegengesetzte Auffassung vertritt Flescher: In seinen Augen gibt es eine „aretaic meta-duty to improve our character" (Flescher 2000, 227f.). 
paradox, wenn jemand eine solche Rettung trotz der Lebensgefahr aufgrund einer individuellen Selbstverpflichtung für seine individuelle Pflicht hält. Mit seiner Äußerung ,I did my duty ' hat Jackowski von seiner Selbstverpflichtung auf einen anspruchsvollen moralischen Selbstentwurf gesprochen, aber nicht von einer allgemeinen Pflicht für alle moralischen Akteure. Auf diese Weise löst sich das Heroismus-Paradox.

3. Warum finden wir Mitläufer unanständig? Warum aber wirken Mitläufer unanständig, wenn es keine allgemeine moralische Pflicht gibt, aktiv und unter Einsatz des eigenen Lebens die Verbrechen eines Unrechtsregimes zu verhindern? Um diese Frage beantworten zu können, muss zunächst untersucht werden, warum Supererogateure moralisch besonders geschätzt und Ultraerogateure sogar bewundert und verehrt werden.

(3.1) Für dieses Phänomen gibt es mehrere mögliche Erklärungen, die hier wiederum nur in aller Kürze angesprochen werden können. (i) Dass Ultraerogateure wegen moralischer Vollkommenheit verehrt werden, kann ausgeschlossen werden, weil Ultraerogateure (anders als fiktive Filmhelden) menschliche Akteure sind, die als solche immer menschliche Schwächen haben. Baron ${ }^{30}$ führt Oskar Schindler als Beispiel dafür an: Er war ein Ultraerogateur - aber er soll auch ein windiger Geschäftsmann und ein untreuer Ehemann gewesen sein (Baron 1998, 64). (ii) Nahe liegt die Antwort, dass wir Ultraerogateure verehren, weil sie moralisch wertvoll handeln. Das kann aber wiederum nur eine notwendige und keine hinreichende Bedingung sein, weil wir schließlich auch dann moralisch wertvoll handeln, wenn wir unsere Pflicht tun, ohne dass wir dafür in besonderer Weise bewundert würden. (iii) Ebenfalls nahe liegt die Antwort, dass wir einen Ultraerogateur wie Jackowski für seine Risikobereitschaft und seinen Mut bewundern. Nun steht außer Frage, dass Jackowski sehr mutig gehandelt hat. Allerdings nehmen Ultraerogateure wie Jackowski anders als Abenteurer die Gefahren oder die Entbehrungen ihres Handelns nicht aus Lust am Risiko in Kauf, sondern um anderen zu helfen. Waghalsige Abenteuer mögen ästhetisch faszinierend und spannend sein, aber solange sie um der bloßen Herausforderung willen geschehen, werden sie anders als Ultraerogateure sicherlich nicht moralisch bewundert. (iv) Dass Ultraerogateure wegen ihres anspruchsvollen moralischen Selbstentwurfs bewundert werden, kann ebenfalls ausgeschlossen

30 Hier wird in Anm. 18 auf ein Interview mit der Witwe Schindlers von 1973 verwiesen. 
werden, weil wir Menschen nicht dafür bewundern, dass sie große Pläne haben, sondern gegebenenfalls nur dafür, dass sie diese Pläne auch realisieren. (v) Damit scheint die Antwort gefunden zu sein: Wir bewundern Ultraerogateure wegen ihres ultraerogativen Handelns. Was aber ist daran bewundernswert, wenn (wie gerade gesagt) die Risikoträchtigkeit von Ultraerogationen nicht der (alleinige) Grund für die Bewunderung von Ultraerogateuren sein kann? Mit Rekurs auf Immanuel Kant lässt sich diese Frage vielleicht beantworten. Mit Kant lässt sich nämlich die ,moralische Freiheit‘ als Voraussetzung für jedes moralische Handeln überhaupt behaupten. Gemeint ist, dass moralische Akteure nur deshalb zu moralischem Handeln in der Lage sind, weil sie grundsätzlich fähig zu sein scheinen, sich gegebenenfalls gegen die eigenen Glücksinteressen für das entscheiden zu können, was sie als das moralisch Richtige eingesehen haben. Schon das ist in vielen Fällen eine groBe Leistung. Die Freiheit zur Supererogation scheint jedoch noch umfassender zu sein: Um supererogativ handeln zu können, muss sich ein Akteur zur Realisierung seines anspruchsvollen moralischen Selbstentwurfs nämlich zusätzlich auch über die vernünftigen Gründe hinwegsetzen können, aus denen manche moralisch wertvolle Handlungen vernünftigerweise keine allgemeine Pflicht sein sollten. Wer das kann, wird zu Recht moralisch besonders geschätzt. Diese moralische Wertschätzung kann sich bis zur Bewunderung und Verehrung steigern. Tatsächlich schätzen wir einen Supererogateur umso mehr für seine Freiheit zur Supererogation, je gewichtiger die Gründe sind, über die er sich hinwegsetzt. Ultraerogateure setzen sich über den besonders gewichtigen (existentiellen) Grund hinweg, dass die Handlung ihren Status als moralischen Akteur gefährden könnte: Es ist diese ultraerogative Freiheit, für die wir sie bewundern. Jackowski wusste, dass seine Handlungen keine allgemeinen Pflichten sind, weil sie lebensgefährlich waren. Er hat die Menschen trotzdem gerettet, weil er sich über die Gründe hinwegsetzen konnte, die er vor sich selbst und vor anderen hätte anführen können, wenn er die lebensgefährlichen Ultraerogationen nicht hätte tun wollen. Und wegen dieser Freiheit zur Ultraerogation wird Jackowski zu Recht als moralischer Held verehrt.

(3.2) Das heißt aber umgekehrt nicht, dass man moralische Akteure wie Bogdanowicz deshalb verachten dürfte, die eine Ultraerogation aus Angst verweigert haben: Das ist ausdrücklich nicht der Fall. Weil es moralisch selbstwidersprüchlich wäre, im Namen der Moral das Selbstopfer moralischer Akteure für andere moralische Akteure zu fordern, müssen Ultraerogationen nämlich in einem radikalen und absoluten Sinne freiwillig 
sein. Daraus folgt nicht nur, dass Ultraerogationen keine Pflicht sein können. Daraus folgt auch, dass sich im Falle einer Verweigerung einer Ultraerogation jede negative moralische Bewertung verbietet. Ultraerogationen bezeichnen die Grenze dessen, was im Namen der Moral gefordert werden kann: Deshalb kann man Ultraerogationen verweigern, ohne mit irgendeiner Missbilligung rechnen zu müssen. Wir bewundern es in höchstem Maße, wenn sich ein Ultraerogateur als Akteur um einer moralisch wertvollen Handlung willen selbst gefährdet - aber es entzieht sich jeder negativen moralischen Bewertung, wenn jemand nicht dazu bereit ist. (i) Das bedeutet erstens, dass sich niemand vor sich selbst moralisch schämen muss, der eine Ultraerogation verweigert. Angemessen wäre lediglich die Art von Scham, die man empfindet, wenn man sich etwas Großes vorgenommen und das dann nicht geschafft hat. (ii) Vor allem aber würde sich jeder selbst unanständig machen, der einen moralischen Akteur dafür kritisiert, dass er nicht ultraerogativ gehandelt hat. Das gilt nicht nur für Akteure, welche die ,Gnade der späteren Geburt' genießen und niemals in einer Situation waren, in der vergleichbares ultraerogatives Handeln moralisch wertvoll gewesen wäre. Auch ein Akteur wie Jackowski (der selbst ultraerogativ gehandelt hat) würde sich mit einem Tadel ein Urteil anmaßen, das ihm nicht zusteht, und in der Tat hat Jackowski Bogdanowicz dann ja auch nicht angeklagt, sondern im Gegenteil entschuldigt. ${ }^{31}$ (iii) Tatsächlich kann man um Ultraerogationen letztlich noch nicht einmal bitten: Ich kann nicht sagen ,Bitte opfere doch Dein Leben, um mich zu retten', ohne mich selbst anrüchig zu machen, weil meine Bitte jemanden in moralisch unzulässiger Weise unter Druck setzen würde.

(3.3) Ganz anders liegt der Fall bei Anstandserogationen. Anstandserogationen sind moralisch wertvolle Handlungen, bei denen man aus anderen Gründen als der Selbstgefährdung des Akteurs als solcher nicht wollen kann, dass sie allgemeine Pflicht sind. Weil es solche Gründe gibt (vgl. die kurze Skizze in Abschnitt 1.3) sind auch Anstandserogationen Ausdruck von supererogativer Freiheit. Auch anstandserogatives Handeln zeugt damit von einem grundsätzlichen Interesse an der Veredelung des eigenen moralischen Selbst. Deshalb werden Anstandserogateure geschätzt und gemocht, wenn sie auch nicht in dem Maße bewundert und verehrt werden wie Ultraerogateure: Das Gefälle erklärt sich dadurch, dass sich die Freiheit zur Anstandserogation über deutlich weniger gewichtige Gründe hinwegsetzen muss als die Freiheit zur Ultraerogation. 
(3.4) Vor diesem Hintergrund lässt sich nun die Frage beantworten, warum Mitläufer unanständig wirken. Als ,Mitläufer ${ }^{`}$ werden Menschen bezeichnet, die es entweder schweigend hinnehmen oder sogar Zustimmung signalisieren, wenn Unrechtsregime schwere Menschenrechtsverletzungen begehen. Beide Spielarten des Mitläufertums wirken unanständig, wenn auch in unterschiedlichen Graden. Um die Unanständigkeit des ,schweigenden Mitläufertums ' zu erklären, ist die Tatsache entscheidend, dass man mit Anstandserogationen sein moralisches Selbst veredeln kann, ohne das Risiko einer Selbstaufgabe einzugehen und ohne den dialektischen Umschwung in die Vernichtung des Selbst befürchten zu müssen, der bei Ultraerogationen ja immer droht. Deshalb kann man aus einer grundsätzlichen Verweigerung von Anstandserogationen nämlich etwas schließen, was man aus der Verweigerung von Ultraerogationen ausdrücklich nicht schließen kann: Aus der grundsätzlichen Verweigerung von Anstandserogationen kann man auf ein grundsätzliches Desinteresse an der Veredelung des eigenen moralischen Selbst rückschließen, was Beobachter unsympathisch und unanständig finden. Nun macht eine Schwalbe noch keinen Sommer, und genauso wirkt eine einzelne Verweigerung einer Anstandserogation noch nicht unanständig. Rückschlüsse auf ein (moralisch anrüchiges) Desinteresse an einer Veredelung des moralischen Selbst lässt nur die grundsätzliche Verweigerung von Anstandserogationen zu, aber ausdrücklich nicht eine einzelne Verweigerung. Nur die grundsätzliche Verweigerung von Anstandserogationen finden wir unanständig, weil nur das auf ein Desinteresse an der Veredelung des moralischen Selbst rückschließen lässt. Man nehme einmal an, dass Jackowski einen Nachbarn gehabt hätte, der gemerkt hätte, was nebenan vor sich ging. Es wäre nicht weiter gefährlich, aber anständig, wenn dieser Nachbar ab und zu einige Lebensmittel oder Kleidungsstücke vorbeibringen würde, um Jackowski unauffällig zu unterstützen. Wenn der Nachbar solche Handlungen jedoch (aus Geiz oder Trägheit oder aus Desinteresse am Leiden anderer) konsequent unterlässt, mag er zwar kein moralisches Vergehen begehen, aber er würde unanständig wirken, weil er durch die Unterlassungen ein grundsätzliches Desinteresse an jeder Veredelung seines moralischen Selbst zum Ausdruck bringen würde.

(3.5) Noch deutlicher wird dieses Desinteresse mit der zweiten Spielart des Mitläufertums signalisiert, wenn ein Akteur sich an den Verbrechen eines Unrechtregimes zwar nicht aktiv beteiligt, aber Zustimmung zeigt, weil er sich davon irgendwelche Vorteile erhofft. In diesem Fall liegt eine Form des Handelns vor, die in der angloamerikanischen Supererogationsforschung als 
,Versto $\beta^{6}$ oder ,Suberogation ${ }^{{ }^{32}}$ bezeichnet wird: Das sind Handlungen, die zwar keine Zuwiderhandlungen gegen ein Gebot darstellen, deren Ausführung aber dennoch moralisch anrüchig zu sein scheint. Ein Beispiel für eine Suberogation findet sich auf einem in der Berliner Ausstellung Topographie des Terrors präsentierten Foto: Es ist grob mit dem Datum ,Ende August 1940'versehen und zeigt eine Menschenmenge auf dem Marktplatz von Ulm, die begeistert klatscht, weil einer jungen Frau wegen einer Beziehung zu einem französischen Kriegsgefangenen die Haare geschoren werden. ${ }^{33}$ Die klatschenden Menschen haben die Frau physisch nicht angerührt, und es ist nicht verboten, auf einem Marktplatz zu klatschen. Damit wäre die Kategorie der Verbotsübertretung unangebracht. Aber mit ihrem schäbigen Handeln um des kleinen Vorteils der Anerkennung durch die Nazi-Herrscher willen haben die Menschen deutlich zum Ausdruck gebracht, dass sie an keiner Veredelung ihres moralischen Selbst interessiert sind. Deshalb finden wir ihr suberogatorisches Handeln mies und unanständig. ${ }^{34}$ Zusammenfassend

32 Zugrunde liegt die Unterscheidung von lässlichen Sünden und Todsünden bei Thomas von Aquin. (i) In der modernen Supererogationsforschung wurde die Kategorie des ,Verstoßes' (offence) eingeführt von Chisholm 1969/1982. Verwiesen wird hier auf Ladd 1957, 108-187, Meinong 1894, 89-92, Schwarz 1934, 35-71. (ii) Seit Driver ist hier in der Regel allerdings von ,Suberogationen“ die Rede. Es heißt: „Suberogatory acts are acts that we ought not to do, but which are not forbidden (we do not have an obligation not to do them)“ (Driver 1992, 291).

33 Das Foto aus einem Nazi-Schmierblatt trägt folgende Unterschrift: „Auf dem Ulmer Marktplatz werden einer 19-Jährigen (...) wegen ihrer Beziehung zu einem französischen Kriegsgefangenen von einem örtlichen Friseur öffentlich die Haare abgeschnitten. Ende August 1940. (...) Das Foto der belustigt zuschauenden Menschenmenge erschien am 28. September 1940 mit der Bildlegende ,Spott und Verachtung standen in den Mienen der Tausenden geschrieben““. Zit. nach Topographie des Terrors. Berlin, August 2018.

34 Um Missverständnisse zu vermeiden: Ich möchte ausdrücklich nicht diejenigen Menschen als unanständige Mitläufer einstufen, die Unanständigkeiten begangen haben, um ihr Leben zu retten. Ein Beispiel findet sich wiederum bei Jackowski. Er berichtet: „I even took in some Jews who had threatened to denounce me and their kinsmen whom I was hiding if I didn't help them." (Lukas 1989, 77). Eine solche Erpressung ist zweifellos für sich genommen unanständig. Dennoch aber sind die Erpresser nicht unanständig, weil sie aus extremer Not gehandelt haben. Ein anderes Beispiel findet sich auf einer Tafel der Berliner Ausstellung Topographie des Terrors. Hier wird die Berliner (Neuköllner) Ärztin Käte Frankenthal zitiert: „Often I couldn’t believe my eyes 
lässt sich also sagen, dass Mitläufertum in beiden Spielarten unanständig wirkt, weil ein grundsätzliches Desinteresse an einer Veredelung des moralischen Selbst zum Ausdruck kommt.

4. Was sollte man anständigerweise tun, wenn man nicht das Zeug zum Helden hat? Wenn Mitläufertum unanständig ist, und wenn gleichzeitig der lebensgefährliche Einsatz gegen die Menschenrechtsverletzungen von Unrechtsregimen keine allgemeine Pflicht sein kann, steht die Frage im Raum, was in Jackowskis Situation tatsächlich moralische Pflicht gewesen wäre? Gibt es so etwas wie eine moralische Pflicht zum Widerstand gegen ein Unrechtsregime? ${ }^{35}$ Natürlich kann diese komplexe Frage hier nicht beantwortet werden; es kann nur um einen kurzen Ausblick gehen.

(4.1) Obgleich die Grenze ${ }^{36}$ nicht immer scharf gezogen werden kann, sollen die Überlegungen durch die grundsätzliche Unterscheidung zwischen aktivem und passivem Widerstand gegliedert sein. In aller hier gebotenen Vorläufigkeit sei unter einem, passiven Widerstand gegen Unrechtsregime die Weigerung verstanden, sich in irgendeiner Weise aktiv an den verbrecherischen Menschenrechtsverletzungen des Regimes zu beteiligen. Es bedarf keiner weiteren Erläuterungen, dass sich aus dem im ersten Abschnitt skizzierten rationalistischen Begriff von ,Pflicht' eine generelle moralische Pflicht zu dieser Form des Widerstandes herleitet: Man kann schließlich offensichtlich nicht wollen, dass sich moralische Akteure aus welchen Grün-

when I saw people I had known for years as Socialists wearing the swastika (...) I don't judge them at all. The swastika was a kind of life insurance, and they wanted to live." Diese Menschen waren keine unanständigen Mitläufer, weil sie ebenso wie die jüdischen Erpresser nackte Angst um ihr Leben hatten.

35 Nach Mellema stellt Widerstand eine Quasi-Supererogation dar: Das sind Handlungen, deren Ausführung preiswürdig (engl. praiseworthy) und deren Unterlassung „tadelnswert“ (engl. blameworthy) sein soll. (i) Wie gezeigt wurde, ist es jedoch nicht ,tadelnswert', keinen Widerstand zu leisten, wenn der Widerstand lebensgefährlich ist. (ii) Mitläufertum ist unanständig. Allerdings ist es umgekehrt auch nicht besonders preiswürdig, wenn man kein Mitläufer ist (auch wenn man dadurch vielleicht auf Vorteile verzichten muss). Deshalb scheint die Kategorie der Quasi-Supererogationen hier keine weiterführende Kategorie zu sein.

36 Das handlungstheoretische Problem, dass sich Aktivität und Passivität (auch im Feld des Widerstandes) nicht immer so strikt unterscheiden lassen, wie es moralphilosophisch wünschenswert wäre, muss hier insgesamt außen vor gelassen werden. Vgl. dazu Birnbacher 1995. 
den auch immer an den Verbrechen eines Unrechtsregimes beteiligen; deshalb gibt es eine generelle moralische Pflicht, jede aktive Beteiligung an Menschenrechtsverletzungen unter allen Umständen zu verweigern. Dem Einwand, dass eine solche Verweigerung lebensgefährlich (und damit ein Kandidat für eine Ultraerogation) sein könnte, möchte ich entgegenhalten, dass faktisch niemandem etwas passiert ist, der den Dienst im KZ verweigert hat. Wiederum findet sich in der Geschichte von Jackowski ein Beispiel für einen Verstoß gegen diese allgemeine Pflicht. Eine der versteckten Frauen hieß Nina Dannenberg. Im Alter von 75 Jahren erinnert sie sich daran, dass ein ukrainischer Schulfreund ihres jüdischen Vaters sich den Nazis als Hilfspolizist angedient und in dieser Rolle ihrem Vater mit einem Gummiknüppel auf den Kopf geschlagen hat, als dieser um die Verschonung seiner alten Eltern von der Deportation bat (Sturrock 2011). Der Hilfspolizist hat einen schweren Pflichtenverstoß begangen, weil er sich mit dem Schlag aktiv an der schweren Menschenrechtsverletzung beteiligt, die eine Deportation zweifellos bedeutet. Im Kontext der angloamerikanischen Supererogationsforschung sollte betont werden, dass dasselbe für Denunziationen gilt, weil Denunziationen hier nämlich (in Anknüpfung an Chisholm ${ }^{37}$ ) als Beispiel für einen ,Verstoß $\beta^{`}$ diskutiert werden. Tatsächlich ist eine Denunziation zumindest dann mehr als nur ein Verstoß, wenn der Denunziant (wie es zu Jackowskis Zeiten zweifellos der Fall war) wissen kann, dass er die Denunzierten schweren Menschenrechtsverletzungen ausliefert. Dann ist eine Denunziation nämlich eine aktive Beteiligung an schweren Menschenrechtsverletzungen und damit ein schwerwiegender Verstoß gegen die moralische Pflicht einer Verweigerung jeder aktiven Teilnahme.

(4.2) Im Feld des aktiven Widerstandes lässt sich grob gewaltsamer und gewaltloser aktiver Widerstand unterscheiden. Wenn der gewaltsame aktive Widerstand gegen Menschenrechtsverletzungen von einer fremden Nation geleistet wird, spricht man vom ,gerechten Krieg' oder von ,humanitärer Intervention'. Es handelt sich faktisch um Kriegshandlungen, die als solche sowohl das Leben der Akteure als auch von Zivilisten gefährden. Nach Schaber ist eine solche Handlung Supererogation, weil das Leben der Akteure gefährdet wird. ${ }^{38}$ Weil bei humanitären Interventionen Zivilisten getötet

$37 \quad$ Vgl. Chisholm 1963/1982, 109f.

38 Zwar fällt der Begriff, Supererogation" bei Schaber nicht. Allerdings ist der Sache nach von Supererogationen (bzw. von Ultraerogationen) die Rede, wenn es heißt: „Sofern Gefahr für das Leben der Kombattanten zu erwarten 
werden können, scheint mir die Dilemma-Diagnose von Dieter Witschen jedoch einleuchtender zu sein. Ein moralisches Dilemma ist eine Situation, in der ein Akteur zwischen zwei Optionen wählen muss, die sich gegenseitig ausschließen, für die aber vergleichbar starke moralische Gründe sprechen, so dass sich der Akteur schuldig macht, gleich welche Option er wählt. Bei humanitärer Intervention besteht die Gefahr, dass Zivilisten getötet werden. Gleichzeitig gibt es bei gravierenden Menschenrechtsverletzungen natürlich sehr gute moralische Gründe für eine Intervention. Deshalb ist gewaltsamer Widerstand ein moralisches Dilemma. ${ }^{39}$ Pragmatisch bedeutet diese moralphilosophische Diagnose, dass Nationen mit Wiedergutmachungsforderungen für zivile Opfer und die Zerstörung von Infrastruktur rechnen müssen, wenn sie sich für eine humanitäre Intervention entscheiden. Aus demselben Grund stellt auch gewaltsamer aktiver Widerstand in Form von Revolutionen, Attentaten und Putschen ein moralisches Dilemma dar. Bekannte Beispiele aus der Zeit der Nazi-Verbrecher wären die Attentate von Eissler oder Claus Graf Schenk von Stauffenberg. Obwohl die Entscheidung für ein Attentat auf den Verbrecher Hitler insgesamt zweifellos die richtige moralische Entscheidung war, hätten sich die Attentäter im Nachhinein doch bei den Familien der Menschen entschuldigen müssen, die sie mit in den Tod gerissen haben, weil sie zufällig auch anwesend waren. Es ist nun einmal das Kennzeichen des moralischen Dilemmas, dass der Akteur nicht handeln kann, ohne in irgendeiner Weise moralisch schuldig zu werden.

(4.3) Bezüglich des gewaltlosen aktiven Widerstandes lassen sich der gewaltlose aktive lebensgefährliche Widerstand und der gewaltlose aktive nicht lebensgefährliche Widerstand unterscheiden. Dass (gewaltloser aktiver) lebensgefährlicher Widerstand Ultraerogation (und damit keine Pflicht) ist, wurde am Beispiel von Jackowski gerade begründet. Andere Beispiele wären die Sabotage in Munitionsfabriken oder das Verteilen von Flugblättern durch die Widerstandsgruppe ,Weiße Rose'. Solcher ultraerogativer Widerstand ist keine allgemeine Pflicht, sondern heldenhaft. Deshalb ist es richtig, dass Straßen nach den Ultraerogateuren benannt und ihre Heldentaten in Filmen nacherzählt werden.

ist, ist also auch bei Humanitären Interventionen Freiwilligkeit gefordert. Konkret bedeutet das, dass die Kombattanten freiwillig darin einwilligen müssen, an solchen Aktionen teilzunehmen" (Schaber 2013, 138).

39 Solche Dilemmata werden als ,Problem der schmutzigen Hände' diskutiert. Vgl. dazu Raters 2013/2016, 76ff. 
(4.4) Es gibt aber auch Formen des gewaltlosen aktiven Widerstandes, die nicht lebensgefährlich sind. Von Jackowskis (fiktivem) Nachbarn war schon die Rede. Vergleichbar kann man sich vorstellen, dass einige der Bauern, bei denen Jackowski einkaufen musste, ihm die Lebensmittel geschenkt haben könnten, weil sie sich gedacht haben, warum ein einzelner Mann so viel davon brauchte. ${ }^{40}$ Ein reales Beispiel wären die bayrischen Katholiken, die sich von ihrem sonntäglichen Kirchgang nicht haben abhalten lassen. Weil es sich um eine nicht lebensgefährliche Form des Widerstandes handelt, steht noch einmal die Frage im Raum, ob solche Formen des Widerstandes gegen Menschenrechtsverletzungen nicht ebenso allgemeine Pflicht wie der passive Widerstand des Verweigerns jeder aktiven Beteiligung an den Menschenrechtsverletzungen sein sollten? Tatsächlich würde das jedoch eine Überdehnung des Pflichtbegriffs bedeuten. Schließlich soll hier ja von einer Pflicht nur die Rede sein, wenn man aus rationalen Gründen wollen kann, dass alle moralischen Akteure in vergleichbaren Situationen eine bestimmte Handlung tun sollen, und das sollte man so kompromisslos wollen können, dass man die Erfüllung der Pflicht einfordern und Zuwiderhandlungen bestrafen wollen würde. Obwohl sie damit zweifellos moralisch wertvoll gehandelt haben, kann man dennoch vernünftigerweise keine allgemeine Pflicht für Bauern wollen, dass sie ihre Lebensmittel an Bedürftige verschenken müssen. ${ }^{41}$ Das gilt erstens, weil man vernünftigerweise nicht wollen kann, dass ein ganzer Berufsstand für seine Arbeit nicht entlohnt wird. Zweitens (das gilt insbesondere in Kriegszeiten) wären die Bauern selbst bald bedürftig, wenn sie allen Bedürftigen helfen müssten. Vergleichbar kann man keine allgemeine Pflicht wollen, aus Demonstrationszwecken in die Kirche gehen zu müssen, weil jeder vernünftige Akteur über seine religiöse Praktiken selbst entscheiden können sollte, und weil man darin auch eine Instrumentalisierung von Religiosität sehen könnte. Es gibt also vernünftige Gründe, aus denen man nicht wollen kann, dass die genannten Formen des (gewaltlosen aktiven) Widerstandes allgemeine Pflicht sein sollen. Weil es sich um nicht lebensgefährlichen Widerstand handeln soll, liegt nicht der

40 In ihren Erinnerungen an ihre Zeit mit Anne Frank berichtet Miep Gies von einem Fleischer und einem Gemüsehändler, die ihr Lebensmittel für die versteckten Familien verschenkt haben. Der Gemüsehändler wurde im März 1944 verhaftet, weil er selbst Verfolgte versteckt hatte (Gies 1987, 111, 170).

41 Die Debatte, ob es sich vielleicht um eine unvollkommene Pflicht handeln könnte, kann hier nicht geführt werden. Vgl. dazu wiederum Raters: Unvollendetes Typoskript. 
Grund einer Gefährdung des Akteurs als solchen für eine Ultraerogation vor. Das alles bedeutet, dass aktiver gewaltloser nicht lebensgefährlicher Widerstand trotz seines moralischen Werts keine allgemeine Pflicht, sondern Anstandserogation ist. Diese Diagnose ist allerdings alles andere als folgenlos: Schließlich lässt sich mit Anstandserogationen ein grundsätzliches Interesse an der Veredelung des moralischen Selbst signalisieren, was auf Beobachter sympathisch und anständig wirkt (vgl. Abschnitt 3.3). Vor diesem Hintergrund hat die Diagnose, dass gewaltfreier, nicht lebensgefährlicher aktiver Widerstand anstandserogativ wäre, nämlich die Funktion eines Ratschlags. Man sollte ab und zu anstandserogativ handeln, wenn man von anderen gemocht und mit sich selbst zufrieden sein will, weil Anstandserogationen zwar keine Pflicht sind, aber immerhin doch auf eine Bereitschaft zur Veredelung des moralischen Selbst rückschließen lassen, was Beobachter mögen. Deshalb ist es anzuraten (wenn auch nicht durch andere einzufordern), dass man (wenn das unter den gegebenen Umständen moralisch wertvoll ist) irgendeine Form von anstandserogativem Widerstand leisten sollte, weil man nur dann von anderen geschätzt wird und mit sich selbst ins Reine kommt. Man wird zwar nicht bestraft, wenn man keinen anstandserogativen Widerstand leistet oder gar unanständige Verstöße begeht. Man wird von anderen jedoch als Mitläufer verachtet, und das ist alles andere als harmlos! Auch das ist ein Resultat der Analyse des Heroismus-Paradoxes.

\section{Literatur}

Archer, Alfred, und Michael Ridge. 2015. „The Heroism Paradox: Another Paradox of Supererogation“. Philosophical Studies 172, 1575-1592. Dordrecht: Springer Science Business Media.

Baron, Marcia. 1987. „Kantian Ethics and Supererogation“. The Journal of Philosophy 84.5, 237-262.

Baron, Marcia. 1998. „Imperfect Duties and Supererogatory Acts“. Jahrbuch für Recht und Ethik. Annual Review of Law and Ethics. Band 6: Altruismus und Supererogation. Hrsg. B. Sharon Byrd, J. Hruschka, Jan C. Joerden, Berlin: Dunker und Humboldt, 57-71.

Benn, Claire. 2019. „Intentions, Motives and Supererogation“. The Journal of Value Inquiry 53. Cham: Springer Nature, 107-123.

Birnbacher, Dieter. 1995. Tun und Unterlassen. Stuttgart: Reclam.

Boghani, P. 2012. „Sen. Daniel Inouye. War Hero“. Global Post, 18.12.2012. Zit. nach http://www.globalpost.com/dispatch/news/regions/americas/united-states/121218/sen-daniel-inouye-war-hero-video. Zugriff 20.12.2012. 
Chisholm, R.M. 1963. „Supererogation and Offence. A Conceptual Scheme for Ethics“. Ratio 5. o.S. Zit. nach ders.: Brentano and Meinong Studies. New York 1982, 98-113.

Colby, A. und W. Damon. 1992. Some Do Care. New York (Free Press).

Curtis, Barry. 1981. „The Supererogatory, The Foolish and the Morally Required“. Journal of Value Inquiry 15, 311-318, The Hague: Martinus Nijhoff Publishers.

Driver, Julia. 1992. „The Suberogatory“. Australian Journal of Philosophy 70.3, 286295.

Flescher, Andrew Michael. 2000. Going beyond the Call of Duty. A Re-examination of the Nature of Heroes, Saints and Supererogation. Diss. Brown University. Zit. UMI Microform Number. 998775. Providence Rhode Island: Bell \& Howell Information and Learning Company. Veröff. auch als Flescher, Andrew. 2003. Heroes, Saints and Ordinary Morality. Georgetown University Press.

Gies, Miep. 1987. Anne Frank Remembered. New York: Simon and Schuster. Zit. als: Meine Zeit mit Anne Frank. Bern: Scherz 1989/Frankfurt a.M.: Fischer 2009.

Groß, Nikolaus. 18. Juli 1944. Zit. nach Topographie des Terrors. Berlin, August 2018.

Gruber, Ruth. 1983/2000. Haven. The Dramatic Story of 1,ooo World War II Refugees and How They Came. University of Michigan: Coward-McCann.

Hale, Susan S. 1991. „Against Supererogation“. In: American Philosophical Quarterly 28.4. 1991, 273-285.

Hallie, Philip. 1979. Lest Innocent Blood Be Shed. New York: Harper.

Hare, Richard Mervyn. 1981. Moral Thinking. Its Levels, Methods and Point. Oxford/New York. Zit. nach ders. 1992. Moralisches Denken. Seine Ebenen, seine Methode, sein Witz. Übers. v. Ch. Fehige und G. Meggle. Frankfurt a.M.

Hare, Richard Mervyn. 1991. „Universal Prescriptivism“. In A Companion to Ethics. Hrsg. V.P. Singer. Oxford. Zit. als ders. 1995. „Zur Einführung. Universeller Präskriptivismus“. In Zum moralischen Denken (2Bd.) Bd. 1. Hrsg. C. Fehige, G. Meggle, 31-54. Frankfurt a.M.: Suhrkamp.

Horgan, Terry, und Mark Timmons. 2010. „Untying a Knot from the Inside Out. Reflexions on the ,Paradox of Supererogation"“. Social Philosophy and Policy 27.2., 29-63. Cambridge Mass.: Cambridge University Press.

Jackson, M.W. 1986. „The Nature of Supererogation“. Journal of Value Inquiry 20.4., 289-296, Dordrecht: Martinus Nijhoff Publishers.

Ladd, John. 1957. The Structure of a Moral Code. A Philosophical Analysis of Ethical Discourse Applied to the Ethics of the Navaho Indians. Cambridge Mass.: Harvard University Press.

Lukas, Richard C., Hrsg. 1989. „Staszeck Jackowski“. In Out of the Inferno. Poles Remember the Holocaust, 76-78. University Press of Kentucky. 
Meinong, Alexius. 1894. Psychologisch-ethische Untersuchungen zur Werth-Theorie. Graz: Verlag: Universitätsbuchhandlungen.

Mellema, Gregory. 1991. Beyond the Call of Duty. New York.

New, Christopher. 1974. „Saints, Heroes and Utilitarians“. Philosophy 49.188. 179189. Cambridge: Cambridge University Press.

Pantel, Nadia. 2018. „Helden willkommen“. In Süddeutsche Zeitung. 28. Mai 2018, 7. München.

Pfannkuche, Walter. 1994. „Supererogation und moralische Verantwortung“. In Umorientierungen, Wissenschaft, Technik und Gesellschaft im Wandel. Hrsg. v. W. König, 73-92. Frankfurt a.M./Berlin/Bern/N.Y./Paris.

Pybus, Elizabeth. 1982. „Saints and Heroes“. Philosophy 57.220. 193-199. Cambridge: Cambridge University Press.

Raters, Marie-Luise. 2013. Zit. ${ }^{2}$ 2016. „Das moralische Dilemma. Antinomie der praktischen Vernunft?" In: Alber - Praktische Philosophie. Freiburg: Alber.

Raters, Marie-Luise. 2020 (i.E.). „Das tue ich nicht, weil es nicht Pflicht ist. Das Argument der Supererogation und sein Unanständigkeitsproblem“. Zeitschrift für philosophische Forschung 74. Hrsg. G. Ernst, C. Rapp. Frankfurt a.M.: Klostermann.

Raters, Marie-Luise. Unvollendetes Typoskript. Das muss ich nicht tun! Das Argument der Supererogation und die Grenzen der Pflicht in der Angewandten Ethik.

Sartre, Jean-Paul. 1946/1973. „L'Existencialisme est un Humanisme. Vortrag Paris 1946“. Im Text zit. nach ders. 1973: „Ist der Existentialismus ein Humanismus?“. In ders.: Drei Essays. Übers. u. hrsg. v. W. Schmiele, 7-51, Zürich.

Schaber, Peter. 2013. „Wann ist der Grund gerecht? Zur Rechtfertigung Humanitärer Interventionen“. In: Die Humanitäre Intervention in der ethischen Beurteilung. Hrsg. H. Busche, D. Schubbe, 127-141, Tübingen.

Schwarz, Ernst. 1934. „Über den Wert, das Soll und das richtige Werthalten“. In: Meinong-Studien II. Graz: Leykam-Verlag.

Stemmer, Peter. 2000. Handeln zugunsten anderer. Eine moralphilosophische Untersuchung. Berlin/New York: De Gruyter.

Sturrock, Staci. 2011. „Heroism Made the Difference for Boynton Beach Retiree Who Survived Holocaust“. In: Palm Beach Post. 18. Mai 2011, o.S.

Tec, Nechema. 1986. When Light Peirced the Darkness. New York.

Urmson, J.O. 1958. „Saints and Heroes“. In Essays in Moral Philosophy. Hrsg. A.I. Melden. Seattle: Washington Press, 198-216.

Witschen, Dieter. 2006. „Mehr als die Pflicht. Studien zu supererogatorischen Handlungen und ethischen Idealen“. In: Studien zur theologischen Ethik. Bd. 114. Hrsg. A. Holderegger. Fribourg (Schweiz). 